\title{
A delay-aware scheduling algorithm for enhancing video services QoS in LTE system
}

\author{
Peng Yang \\ China Academy of \\ Telecommunication Research \\ of MIIT, China \\ yangpeng@catr.cn \\ Dapeng Wu \\ Chongqing University of Posts \\ and Telecommunications, \\ China \\ wudapengphd@gmail.com
}

\author{
Junjie Yan \\ Chongqing University of Posts \\ and Telecommunications, \\ China \\ cqupt2013yjj@sina.com \\ $\mathrm{NaShu}$ \\ Chongqing University of Posts \\ and Telecommunications, \\ China \\ shuna@cqupt.edu.cn
}

\begin{abstract}
Multimedia services are carried by data packet in LTE (Long Term Evolution, LTE) system, such as voice, video, and so on. Quality of Service in multimedia services is essential to user experience. In this paper, we present a packet scheduling algorithm to improve the performance of video traffic. By considering the number of packet loss and packet delay in the buffer, the priority of each user can be adjusted dynamically to reduce the packet loss rate and maximize system throughput. The results show that compared with the current typical packet scheduling algorithm, the proposed algorithm can significantly improve the packet loss rate, fairness, throughput and other performance.
\end{abstract}

\section{Categories and Subject Descriptors}

C. 2 [COMPUTER-COMMUNICATION NETWORKS]: Miscellaneous

\section{General Terms}

Algorithms,Experimentation,Performance

\section{Keywords}

Long Term Evolution; packet scheduling; Quality of Service; delay

\section{INTRODUCTION}

In order to satisfy the demand of the growing data services in communications system, 3GPP (The 3rd Generation Partnership Project, 3GPP) started the LTE technology standardization work in 2004. The overall goals of the
LTE are determined for high rate, low delay and packetoptimized radio-access technology. LTE system uses OFDM (Orthogonal Frequency Division Multiplexing, OFDM) and MIMO (Multiple Input Multiple Output, MIMO) technology as an important feature in wireless network evolution. Its peak rate is downlink $100 \mathrm{Mbit} / \mathrm{s}$ and uplink 50Mbit / $\mathrm{s}$, improving the quality of the service of cell edge users [1].

As is known to all, high-quality video services have high requirements for network bandwidth and terminal transmission rate. It needs advanced video compression and coding techniques and transmission and switching technologies, also including more stringent packet scheduling algorithms, whose main goal is to reduce the packet loss rate, maximize system throughput, ensure fairness among users and guarantee QoS (Quality of Service, QoS) requirements of different traffic $[2]$.

In order to maximize system throughput or maintain fairness between users, the main principle of the conventional scheduling algorithm is aware of the real-time channel state, such as the maximum carrier to interference ratio ( $\max \mathrm{C} / \mathrm{I}$ ) algorithm, the RR (Round Robin, RR) algorithm, PF (Proportional Fair, PF) algorithm and so on. However, the above algorithm does not think many other factors about influencing the QoS of video services. Therefore, the effect of video transmission services can not satisfy the actual demand [3].

\section{RELATED WORK}

The M-LWDF (Modified Largest Weighted Delay First, M-LWDF) algorithm and EXP (Exponential, EXP) algorithm are analyzed in [4]. They are not only considering the instantaneous channel quality perceived by the user, but also the packet queuing delay, so achieving a good balance in the system throughput and fairness among users. Considering the lack of the user fairness in M-LWDF algorithm, [5] is proposed based on linear and non-linear priority improved M-LWDF algorithm, in order to achieve the purpose of the user to enhance the fairness. Because the packet loss rate is very sensitive in video services, [6] proposed DPS (DelayPrioritized Scheduling, DPS) algorithm, based on the information of the packet delay and instantaneous SNR. In this algorithm we can see the longer delay user who is in the packet buffer can be assigned the best RB, until all RBs are 
allocated. The system throughput performance of the DPS algorithm is better. It reduces the packet loss rate effectively, but the algorithm mainly considers the packet delay information. So that some poor channel conditions users get more services, limiting the performance of the system.

A priority scheme of voice user equipment in LTE is introduced to the semi-persistent resource scheduling mechanism for the radio resource in [7]. This paper comes up with a VoLTE radio resource scheduling scheme which is based on the priority of users. The scheme can avoid transporting the radio resource scheduling signaling data frequently and competing limited shared wireless resources by voice data. It is not only improving the utilization efficiency of radio resources and scheduling efficiency, but also increasing user capacity and user satisfaction.

[8] proposed a mechanism which is suitable for the adaptive resource allocation of the multicast broadcast SFN transmission mode in LTE system. The mechanism according to the proportion of business and the corresponding sub-frame SINR threshold adaptively realizes sub-frame allocation between multicast traffic and unicast traffic. This algorithm ensures the multicast traffic rate, improves the unicast traffic throughput, and optimizes the system radio resources.

[9] proposed a cross-layer design scheme based video traffic in the quality of the user experience. The scheme uses cross-layer architecture and the mapping model of hyperbolic tangent function curve, reflecting the peak signal to noise ratio and mean opinion score relationships. Furthermore, on this basis this article put forward prediction function of the average score based on the user experience quality, achieving the best allocation of resources between users.

Above scheduling algorithms balance the throughput and fairness issues from packet delay and channel conditions. However, in the video traffic applications, not only should we consider the above problem, packet loss rate also should be thought as a significant impact on system performance.

\section{DELAY AWARE SCHEDULING ALGO- RITHM}

ENodeB is the base station which specifies a buffer to each user. All kinds of packet services get into the buffer using the principle of FIFO (First In First Out, FIFO). At the same time, they are set the corresponding time stamp and wait for transmission. In each TTI (Transmission Time Interval, TTI), one of the users is chose to transfer data according to packet scheduling algorithms.

For LTE system carries video services faced with many problems, this paper proposes a delay-sensitive proportional fairness algorithm, called DA-PF (Delay Aware-Proportional Fair, DA-PF) algorithm. In order to reduce system loss rate and maximize system throughput, this algorithm is mainly aimed at the packet transmission time which is about to expire. Furthermore, it will ensure the fairness of the system according to the number of packet loss in the queue.

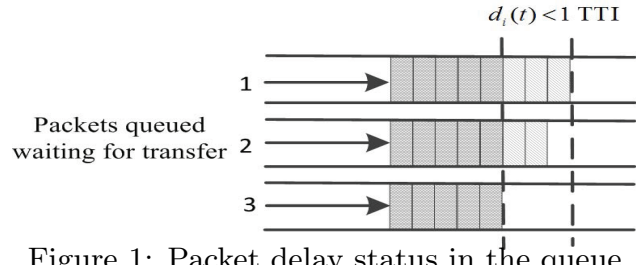

Figure 1: Packet delay status in the queue
According to the basic principle of the LTE system packet scheduling, there exist many discarding packets a packet in a scheduling interval. As shown in the Fig 1, Due to the strong delay sensitivity in packet video services, the system assigns higher priority to high delay packet. As the algorithm allocates resources taking into account the rate sensitivity, the user in the better channel conditions can get more scheduling opportunities. Therefore, in order to meet the QoS requirements of video data stream and ensure fairness among users, the algorithm considers the user's packet delay, packet loss rate and instantaneous rate as scheduling judgment.

Scheduling emergency degree is determined according to the packet delay in queue. For example, at each scheduling time interval, the remaining time of user $i$ is calculated from HOL (Head Of Line, HOL) packet delay to the maximum packet delay at $t$ time.HOL packet delay was proposed by M-LWDF. The bigger HOL became, the higher priority improved.

$$
d_{i}(t)=T_{i}-W_{i}(t) i \forall \text { user }
$$

$T_{i}$ is the maximum packet delay of user $i, W_{i}(t)$ is the HOL packet delay of user $i$ at $t$ time.

When user's channel conditions are approximately same, the traditional method uses the instantaneous channel conditions and average throughput to ensure the long-term fairness of the system [11]. The proposed algorithm which can improve the fairness of the system in the short-term takes into account the number of lost packets in the different queues and specific time, and avoids the packet buffer too long and causing packet loss. To calculate the amount of packet loss in the queue, eNodeB sets the appropriate counter for each queue.

$N V_{i}(t)$ indicates the number of user $i$ 's packet loss at the $t$ time. When $T_{i} \leq W_{i}(t)$, data packets start losing. $\overline{N V(t)}$ is the average value of the packet loss in $\mathrm{N}$ queues.

$$
\overline{N V(t)}=\frac{1}{N} \sum_{i=1}^{N} N V_{i}(t)
$$

According to the packet loss information in the queue to adjust the priority of the queue improves the short-term fairness of the algorithm.

The counter accumulates the number of the packet loss for a long-term, so the data information of recent packet loss can not reasonably be reacted. In particular, when the cumulative number of the packet loss is large in a queue, the factor of algorithm $N V_{i}(t) / \overline{N V(t)}$ plays a great impact on the scheduling algorithms. This queue may get sustained scheduling service, affecting the fairness of the system. Therefore, the counter will initialize the user who has completed the scheduling. When user $i$ is scheduling service at the $t$ time, packet loss statistics of the corresponding queue becomes zero. That is to say $N V_{i}(t)=0, i=j$.

In summary, the proposed scheduling algorithm determine the degree of emergency according to the packet delay information of the queue, so users with larger delay will obtain higher priority and based on the instantaneous channel condition, the system throughput will be maximized. At the same time, by considering the packet loss information of the queue, the user's fairness is improved. Specific scheduling algorithms are shown in eq.(3) and eq.(4). 


$$
\begin{gathered}
j=\arg \max _{i}\left\{\frac{N V_{i}(t)}{\overline{N V(t)}} \frac{r_{i}(t)}{\left[\overline{R_{i}(t)}\right]^{\beta}}\right\} \\
\beta=\min \left\{\frac{\left\lfloor d_{i}(t)\right\rfloor}{\mathrm{TTI}}, \frac{d_{i}(t)}{T_{i}}\right\}
\end{gathered}
$$

$r_{i}(t)$ is the instantaneous rate and $R_{i}(t)$ is the average rate of the user at the $t$ time. TTI is the packet scheduling interval, and $\left\lfloor d_{i}(t)\right\rfloor$ is down to the nearest integer arithmetic. When $d_{i}(t)<1 \mathrm{TTI},\left\lfloor d_{i}(t)\right\rfloor=0$ and $\beta=0$. At this time, the user $i$ which is about to be lost in the queue gets higher priority, and according to the user's instantaneous channel conditions, the user with larger instantaneous rate will be scheduling prior; When $d_{i}(t) \geq 1 \mathrm{TTI}$, exponential factor $d_{i}(t) / \mathrm{TTI} \geq 1, d_{i}(t) / \mathrm{T}_{i}<1$ and $\beta=d_{i}(t) / \mathrm{T}_{i}$. That is to say packets with larger delay will obtain higher priority.

\section{NUMERICAL RESULTS}

In order to verify the proposed algorithm, this section uses the LTE-Sim simulation platform [11] to simulate, and the performance of M-LWDF, EXP and DPS algorithm will be compared with the proposed algorithm.

Due to the requirements of QoS in video services, the system performance parameters include the packet loss rate, fairness, throughput and delay, etc. Users move at the speed of $3 \mathrm{~km} / \mathrm{h}$ within the range of the base station, and other simulation parameters are shown in Table 1.

Table 1: Simulation /Numerical parameters

\begin{tabular}{lc}
\hline Simulation & Numerical \\
parameters & \\
\hline Duplex mode & FDD \\
Radius & $10 \mathrm{Km}$ \\
Bandwidth & $180 \mathrm{~s}$ \\
Simulation time & $1 \mathrm{~ms}$ \\
Scheduling period & 50 \\
Resource blocks & $10,15,20,25$, \\
The number of users & $30,35,40$ \\
& Video \\
Transmission Type & streaming \\
& $242 \mathrm{kbps}$ \\
Video rate & M-LWDF,EXP, \\
Downlink scheduling & DPS,DA-PF \\
algorithm &
\end{tabular}

The small number of users with longer average delay gets more system resources in DA-PF scheme, and the average waiting time of newly arriving packets are increased. DP$\mathrm{S}$ algorithm has a similar principle, but the DPS algorith$m$ mainly considers the delay information. The users with better channel conditions will reduce the opportunities of scheduling and increase the packet loss rate, while the user$\mathrm{s}$ with better channel conditions get more opportunities of scheduling in the DA-PF algorithm, so that the packet loss rate is reduced. The performance curve is shown in Figure 2. The simulation results show that the packet loss rate in DA-PF algorithm is lower $60 \%$ than M-LWDF, EXP and DPS.

In the M-LWDF and EXP algorithm, if users do not get service for a long time, regardless of the current channel conditions of users. Users are all allocated fewer RB, which
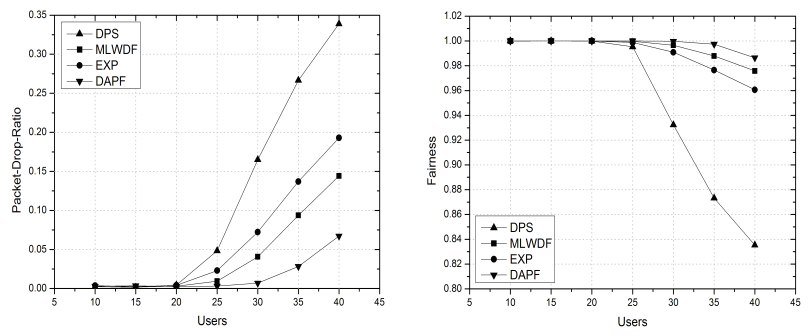

Figure 2: Packet Drop RatioFigure 3: Fairness of Video of Video Service

Service

leads to a higher loss rate. However, the M-LWDF and EXP algorithm also consider the HOL delay information, improving the weight of longer packet delay, so that the packet loss rate of M-LWDF and EXP algorithm is lower than DPS, as shown in Figure 2. In addition, M-LWDF is slightly better than the EXP / PF algorithm. When the HOL delay of user is approximately the same, the performance EXP / PF algorithm converges to PF.

Fairness performance curves of different scheduling algorithms are shown in Figure 3. We can see that DA-PF algorithm has better fairness. The reason is that DPS algorithm mainly considers the packet delay information, while DA$\mathrm{PF}$ algorithm not only considers the packet delay information, but also takes into account the instantaneous channel conditions. Shown in Figure 3, the fairness of DA-PF algorithm compared with M-LWDF and EXP-PF algorithm has some degree of improvement, which because DA-PF algorithm considers the balance the number of packet loss in each queue. When the number of packet loss is increased, its priority of the queue is also improved. The result is that the fairness among users is enhanced. At the same time, users with longer delay are considered first in DA-PF algorithm, so that the performance of packet delay is decreased while fairness among users is raised.
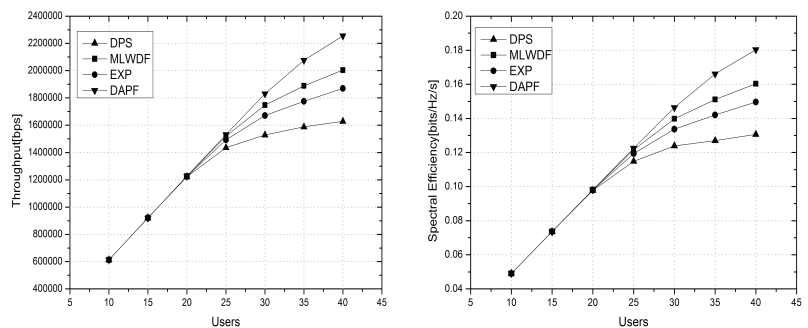

Figure 4: Throughput ofFigure 5: Spectral efficiency Video Service of Video Service

The system throughput of different algorithms can be shown in Figure 4. As we can see, Comparison with the system throughput, the throughput performance of DA-PF algorithm is superior to the other three algorithms, as it increases the weight of instantaneous channel conditions. when $d_{i}(t)<1 \mathrm{TTI}$, the users with better channel conditions get more opportunities of scheduling and its system throughput is enhanced significantly. The HOL delay is considered by M-LWDF and EXP/PF algorithms, so there is a similar throughput. DPS algorithm does not consider the impact of the instantaneous channel conditions in the physical layer, so the performance of system throughput is worst.

Spectral efficiency of radio resources is an important performance index. Figure 5 is the spectral efficiency performance curve of the DA-PF algorithm and other algorithms. 
Compared with the spectral efficiency of M-LWDF, EXP$\mathrm{PF}$ and DPS algorithms, DA-PF algorithm is improved by $10 \%, 15 \%$ and $20 \%$.

Figure 6 shows the packet delay performance curve. DA$\mathrm{PF}$ algorithm, compared with the packet delay of M-LWDF, EXP and DPS algorithms have raised the certain level. Meanwhile, with the increasing number of users in the cell, the packet delay is also increasing. In different scheduling algorithms, DPS algorithm mainly considers the packet delay information, so its delay is less. While the DA-PF algorithm not only considers the packet delay information, but also takes into account the instantaneous channel conditions. The scheduling opportunity of the worse channel conditions users is reduced, leading to increase the waiting time and average delay.

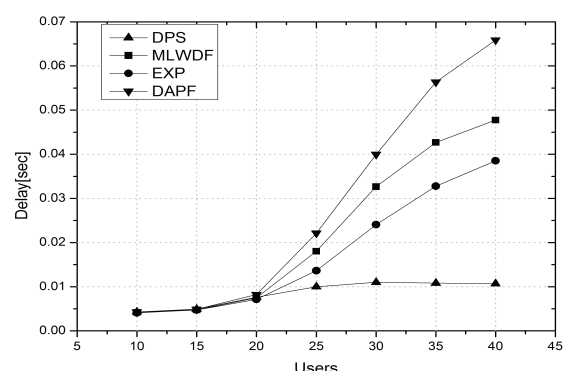

Figure 6: Delay of Video Service

Note that all the implementations of scheduling rules are the same. Firstly, the priority of all active flows is calculated on each RB. Then, the scheduler assigns RB to the highest priority user, in order that scheduling rules take advantage of multi-user frequency diversity characteristics. Scheduling algorithm is designed primarily considering channel conditions, delay, packet loss rate and other factors. Table 2 summarizes the characteristics of several scheduling algorithms. DA-PF algorithm considers the packet loss information, which significantly reduces the loss rate of system.

Table 2: Characteristics of different scheduling algorithms

\begin{tabular}{lccc}
\hline $\begin{array}{l}\text { Scheduling } \\
\text { parameters }\end{array}$ & $\begin{array}{c}\text { Channel } \\
\text { aware }\end{array}$ & $\begin{array}{c}\text { Delay } \\
\text { aware }\end{array}$ & $\begin{array}{c}\text { Packet loss } \\
\text { rate aware }\end{array}$ \\
\hline M-LWDF & Yes & Yes & No \\
EXP & Yes & Yes & No \\
DPS & No & yes & No \\
DA-PF & Yes & Yes & yes \\
\hline
\end{tabular}

\section{CONCLUSIONS}

This paper studies the problem of LTE downlink packet scheduling system, proposing a delay-aware packet scheduling algorithm. The algorithm takes into account the length of packet delay to the maximum delay and the amount of packet loss in the short term. Then, this paper analyzes the M-LWDF, EXP, DPS and DA-PF algorithm. Simulation results show that the delay performance of DA-PF algorithm is lower than other three algorithms; While in terms of fairness, throughput, and packet loss rate, DA-PF algorithm is significantly better than the other three algorithms. Future studies will consider different application environments. In addition, a compensation mechanism will be considered to reduce the delay of the system.

\section{ACKNOWLEDGMENTS}

This work is supported in part by the National Natural Science Foundation of China (61371097), Chongqing Natural Science Foundation (Grant No. CSTC2013JJB40001, CSTC2013JJB40006, CSTC2011JJA40043, CSTC2014JCYJA 40039), the Project of Chongqing Education commission (KJ1400402), the Foundation of Chongqing University of Posts and Telecommunication (A2012-93).

\section{REFERENCES}

[1] Iturralde M, Wei A, Yahiya T A, et al, "Resource allocation for real time services using cooperative game theory and a virtual token mechanism in LTE networks," IEEE Press on Consumer Communications and Networking Conference (CCNC), Las Vegas, pp. 879-883, Jan 2012.

[2] Cicconetti C, Erta A, Lenzini L, et al, "Performance evaluation of the IEEE 802.16 MAC for QoS support," IEEE Transactions on Mobile Computing, vol. 6, no. 1, pp. 26-38, Jan. 2007.

[3] Pahalawatta P V, Katsaggelos A K, "Review of content-aware resource allocation schemes for video streaming over wireless networks," Wireless Communications and Mobile Computing, vol. 7, no. 2, pp. 131-142, Feb. 2007.

[4] Andrews M, Kumaran K, Ramanan K, et al, "Providing quality of service over a shared wireless link," IEEE Communications Magazine, vol. 39, no. 2, pp. 150-154, Feb. 2001.

[5] Dapeng W, Haisheng Y, Renze L. et al, "Fast fairness packet scheduling algorithm for real-time service," Journal of Chongqing University of Posts and Telecommunications : Natural Science Edition, vol. 25, no. 1, pp. 85-89, Feb. 2013.

[6] Sandrasegaran K, Mohd Ramli H A, Basukala R, "Delay-Prioritized Scheduling (DPS) for real time traffic in 3GPP LTE system," IEEE Press on Wireless Communications and Networking Conference (WCNC), Sydney, pp. 1-6, Apr. 2010.

[7] K. Sambale, K. Klagges, R. R. Rezai, "Persistent resource allocations for VoIP traffic in packet-switching mobile cellular networks," Transactions on Emerging Telecommunications Technologies, vol. 21, no. 8, pp. 750-759, Nov. 2010.

[8] Tiankui Z, Aoxue J, Chunyan F, "Adaptive resource allocation scheme for MBSFN transmission in LTE," Journal of Xidian University (Natural Science Edition), vol. 39, no. 5, pp. 126-131, Apr. 2013.

[9] Ju Y, Lu Z, Ling D, et al, "QoE-based cross-layer design for video applications over LTE," Multimedia Tools and Applications, vol. 72, no. 2, pp. 1093-1113, Mar. 2013.

[10] Ning X, Guillaume V, Wen Z, et al, "A dynamic PF scheduler to improve the cell edge performance," IEEE Press on Vehicular Technology Conference, Calgary, pp. 1-5, sept 2008.

[11] Piro G, Grieco L A, Boggia G, et al, "Simulating LTE cellular systems: an open-source framework," IEEE Transactions on Vehicular Technology, vol. 60, no. 2, pp. 498-513, Nov. 2013. 